\title{
Modeling of the Aircraft Landing Behavior for Runway Excursion and Abnormal Runway Contact Analysis
}

\author{
Chong Wang* and Florian Holzapfel ${ }^{\dagger}$ \\ Institute of Flight System Dynamics, Boltzmannstraße 15, 85748 Garching, Germany
}

\begin{abstract}
For the quantification of occurence probabilities of incidents during flight operations, a model-based approach using recorded flight data as inputs has been developed. This work presents a model of the aircraft that is tailored to investigate two frequent accident types during landing: Runway Excursion and Abnormal Runway Contact. The focus of the work is the modeling of the landing gear forces as they play an essential role during this particular phase of flight.
\end{abstract}

\section{Nomenclature}

$\begin{array}{ll}B & \text { Pacejka coefficient } \\ C_{L} & \text { Lift coefficient } \\ C_{n} & \text { Yaw moment coefficient } \\ c & \text { Damper contant } \\ \overrightarrow{\boldsymbol{F}} & \text { Force vector } \\ k & \text { Spring constant } \\ J & \text { Moment of inertia } \\ m & \text { Mass } \\ n_{V} & \text { Speed dependency exponent } \\ q & \text { Pitch rate } \\ S & \text { Reference area } \\ T & \text { Engine thrust } \\ \alpha & \text { Angle of attack } \\ \delta_{S} & \text { Slats deflection } \\ \zeta & \text { Rudder deflection } \\ \xi & \text { Aileron deflection } \\ \text { Subscripts }\end{array}$

$\begin{array}{ll}C & \text { Pacejka coefficient } \\ C_{l} & \text { Roll moment coefficient } \\ C_{Q} & \text { Lateral force coefficient } \\ D & \text { Pacejka coefficient } \\ g & \text { Gravitational constant } \\ k_{i} & \text { Constants for acceleration modeling } \\ j & \text { Landing gear index } \\ m & \text { Total number of landing gears } \\ n_{\rho} & \text { Air density dependency exponent } \\ r & \text { Yaw rate } \\ s & \text { Half wingspan } \\ V & \text { Velocity } \\ \beta & \text { Angle of sideslip } \\ \delta_{S p} & \text { Spoiler deflection } \\ \mu & \text { Friction coefficient } \\ \rho & \text { Air density }\end{array}$

$C_{D} \quad$ Drag coefficient

$C_{m}$ Pitch moment coefficient

$\bar{c} \quad$ Mean aerodynamic chord

E Pacejka coefficient

$k$ Oswald factor

$i \quad$ Engine index

$\vec{M} \quad$ Moment vector

$n \quad$ Total number of engines

$p \quad$ Roll rate

$\vec{r} \quad$ Displacement vector

$s \quad$ Slip

$z \quad$ Landing gear compression

$\delta_{F} \quad$ Flaps deflection

$\delta_{T} \quad$ Throttle lever

$\eta \quad$ Elevator deflection

$\omega \quad$ Wheel angular speed

A Aerodynamic frame

$B \quad$ Body-fixed frame

G Gravitation

$L \quad$ Landing gear

$L \quad$ Longitudinal direction

$N \quad$ Normal direction

$O$ North-East-Down frame

$Q \quad$ Lateral direction

$P \quad$ Propulsion

$T$ Tire

$W \quad$ Wheel

Superscripts

A Aerodynamic reference point $G \quad$ Center of gravity

$P \quad$ Propulsion reference point $G S$ Groundspeed

L Landing gear reference point opt Optimum

*Ph. D. candidate, Email: chong.wang@tum.de

${ }^{\dagger}$ Full professor, Email: florian.holzapfel@tum.de, AIAA Associate Fellow 


\section{Introduction}

The landing and approach phase is the most critical phase during a commercial flight, although accounting for only a small portion of the total flight time. ${ }^{2}$ Two of the most common accident types occurring during the landing are Runway Exursion (RE) and Abnormal Runway Contact (ARC). According to the ICAO Safety Management Manual, ${ }^{3}$ each state as well as each service provider in the aviation transport industry shall define an Acceptable level of Safety Performance (ALoSP), which is a target for the safety of the flight operation in the respective organization. The idea is to compare the current safety performance with the safety target in order to identify and close the gaps. The current level of safety, however, is usually not easily to be determined simply because counting accidents from operational data does not provide the desired results since accidents rarely happen or at least rarely happen within a specific organization. Thus, a model-based method has been developed to quantify the occurrence probabilities of incidents and accidents. ${ }^{4}$ The idea is that, even though accidents rarely happen, the exceedance of certain threshold values of the contributing factors occur frequently. However, one factor being outside of the usual range typically does not lead to an accident due to the robustness of the aviation system. By propagating distributions of contributing factors through a model of the aircraft tailored to specific types of accidents, ${ }^{5}$ one is able to quantify the probability of exceedance of characteristic values that provide statements about whether a specific accident occurred or not.

The models that have been developed so far all focus on the longitudinal direction during the landing. They account for runway overrun, tail strike and hard landing. ${ }^{6}$ The model described in this work was developed to cover additional types of accidents that involve the lateral motion of the aircraft. Types of accidents that can be evaluated with this model include runway veer-off, wingtip strike, engine strike and horizontal tail plane (HTP) strike.

\section{Modeling}

Within the model, the aircraft is considered to be a rigid body with six degrees of freedom (6-DoF). Four types of forces and moments act on the aircraft:

- Gravitation

- Aerodynamics

- Propulsion

- Landing gear forces

As the landing gear force is one of the main drivers of the considered accidents, it will be the main focus of the present work.

\section{A. Gravitation}

Since neither the WGS84 altitude nor the latitude and longitude change significantly during the landing, the gravitation force $\overrightarrow{\boldsymbol{F}}_{G}$ is considered to be a constant vector pointing in the direction of the $\mathrm{z}$ axis of the North-East-Down (NED, index $O$ ) frame $O$ acting at the center of gravity $G$.

$$
\left(\overrightarrow{\boldsymbol{F}}_{G}^{G}\right)_{O}=m\left[\begin{array}{c}
0 \\
0 \\
-g
\end{array}\right]_{O}
$$

\section{B. Aerodynamics}

The aerodynamics model used here is a linear model due to the assumption that the aircraft will not have significant deviations from the linearized state during landing. The angle of attack $\alpha$ and the angle of sideslip $\beta$ should be in the nominal range as well as the body rates $p$ (roll), $q$ (pitch) and $r$ (yaw).

As control inputs, the primary flight control surfaces are used. They include the aileron $\xi$, the elevator $\eta$ as well as the rudder $\zeta$. In addition, the flaps $\delta_{F}$, the slats $\delta_{S}$ and the spoilers $\delta_{S p}$ are considered in the model. 
The coefficients to be obtained are the force coefficients that include the drag coefficient $C_{D}$, the lateral force coefficient $C_{Q}$ and the lift coefficient $C_{L}$ as well as the aerodynamic moment coefficients that include the roll moment $C_{l}$, the pitch moment $C_{m}$ and the yaw moment $C_{n}$ coefficient. The aerodynamic forces $\overrightarrow{\boldsymbol{F}}_{A}$ and moments $\overrightarrow{\boldsymbol{M}}_{A}$ can then be computed using these coefficients:

$$
\begin{aligned}
& \left(\overrightarrow{\boldsymbol{F}}_{A}^{A}\right)_{A}=\frac{1}{2} \rho V_{A}^{2} S\left[\begin{array}{c}
-C_{D} \\
C_{Q} \\
-C_{L}
\end{array}\right] \\
& \left(\overrightarrow{\boldsymbol{M}}_{A}^{A}\right)_{B}=\frac{1}{2} \rho V_{A}^{2} S\left[\begin{array}{c}
s \cdot C_{l} \\
\bar{c} \cdot C_{m} \\
s \cdot C_{n}
\end{array}\right]
\end{aligned}
$$

where $\rho$ is the air density, $V_{A}$ is the aerodynamic velocity (true airspeed) and $S$ is the wing reference area. $s$ is the half of the wingspan and $\bar{c}$ is the mean aerodynamic chord (MAC). The aerodynamic forces and moments act at the aerodynamic reference point $A$. Using the drag, side force and lift coefficient, the aerodynamic forces are expressed in the aerodynamic frame $A$, the moments are expressed in the body-fixed frame $B$.

\section{Aerodynamics Force Coefficients}

For the lift coefficient, a linear approach with respect to the angle of attack, the elevator deflection and the spoiler deflection is used:

$$
C_{L}=C_{L 0}+C_{L \alpha} \cdot \alpha+C_{L \eta} \cdot \eta+C_{L \delta_{S p}} \cdot \delta_{S p}
$$

For the drag coefficient, a quadratic polar is used:

$$
C_{D}=C_{D 0}+k \cdot\left(C_{L}^{2}-C_{L 0}\right)+C_{D \beta} \cdot \beta+C_{D \delta_{S p}} \cdot \delta_{S p}
$$

The coefficient for the lateral force is also influences by the deflection of the rudder:

$$
C_{Q}=C_{Q \beta} \cdot \beta+C_{Q \zeta} \cdot \zeta
$$

For each configuration, i.e. depending on the position of the flaps and slats as well as the extension of the landing gear, the coefficients have to be obtained separately. However, as only the last portion of the landing is considered, configuration changes do not need to be taken into account.

\section{Aerodynamics Moment Coefficients}

$$
\begin{gathered}
C_{l}=C_{l \beta} \cdot \beta+C_{l p} \cdot p+C_{l r} \cdot r+C_{l \xi} \cdot \xi+C_{l \zeta} \cdot \zeta \\
C_{m}=C_{m \alpha} \cdot \alpha+C_{m \eta} \cdot \eta+C_{m q} \cdot q+C_{m \delta_{S p}} \cdot \delta_{S p} \\
C_{n}=C_{n \beta} \cdot \beta+C_{n p} \cdot p+C_{n r} \cdot r+C_{n \xi} \cdot \xi+C_{n \zeta} \cdot \zeta
\end{gathered}
$$

\section{Propulsion}

The propulsion force is described using the rotational speed of the low pressure shaft $\omega_{N 1, i}$ of each engine $i$ out of a total of $n$ engines as a proportional scale factor that obtains the thrust from the maximum available thrust of the engine $T_{\max , i}$ at a given air density $\rho$, as the available thrust on a engine reduces as the density of the air decreases. The reference air density $\rho_{0}$ is used along with an engine parameter $n_{\rho}$ as the exponent. The dependency on the aircraft's speed is considered by introducing the exponent $n_{V}$, using $V_{0}$ as a reference speed. The force of each engine act at the reference point of each engine $P_{i}$. It is assumed that the thrust always acts in the direction of the $\mathrm{x}$ axis of the body-fixed frame (B). A reference point for propulsion forces $P$ is defined for the forces of each engine. 


$$
\left(\overrightarrow{\boldsymbol{F}}_{P}^{P}\right)_{B}=\sum_{i=1}^{n}\left(\overrightarrow{\boldsymbol{F}}_{P, i}^{P_{i}}\right)_{B}=\sum_{i=1}^{n}\left[\begin{array}{c}
T_{i} \\
0 \\
0
\end{array}\right]_{B}=\sum_{i=1}^{n}\left(\frac{\rho}{\rho_{0}}\right)^{n_{\rho}}\left(\frac{V}{V_{0}}\right)^{n_{V}}\left[\begin{array}{c}
\omega_{N 1, i} \cdot T_{\text {max }, i} \\
0 \\
0
\end{array}\right]_{B}
$$

For the moments, the lever arm $\left(\overrightarrow{\boldsymbol{r}}^{R P_{i}}\right)$ from the reference point to each engine has to be considered.

$$
\left(\overrightarrow{\boldsymbol{M}}_{P}^{P}\right)_{B}=\sum_{i=1}^{n}\left(\overrightarrow{\boldsymbol{r}}^{R P_{i}}\right) \times\left(\overrightarrow{\boldsymbol{F}}_{P, i}^{P_{i}}\right)_{B}
$$

\section{Landing Gear}

The landing gear forces represent a central part of the current work. For the landing gear, a model containing a mass, a spring and a damper is used, as shown in Fig. 1.

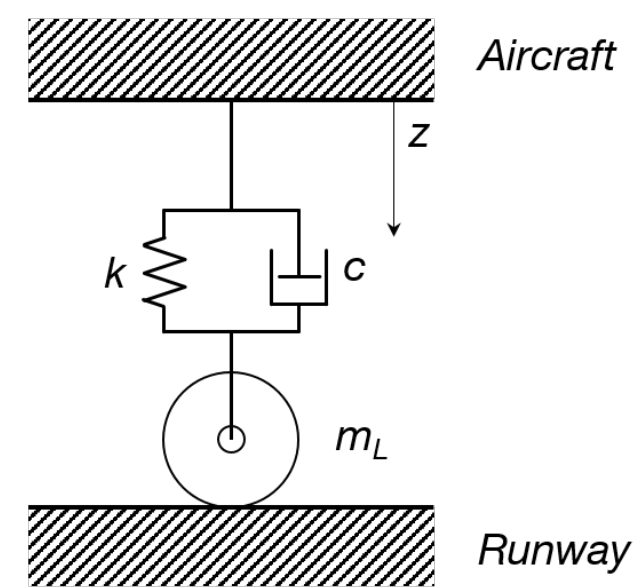

Figure 1. The mass-spring-damper model used for the landing gear.

While the tire itself also has spring and damping characteristics, it is always possible to reduce the model to the one shown in Fig. 1. The spring characteristic $k$ and the damping characteristic $c$ do not necessarily have to be constant, they can also depend on the deflection $z$, i.e. $k=k(z)$ and $c=c(z)$. The part of the landing gear that is below the spring and the damper has a mass of $m_{L}$.

The forces acting on the landing gear can be split into the three components:

- Force acting perpendicular on the contact surface between the tire and the runway, referred to as the normal force $F_{L, N}$.

- Force acting parallel to the contact surface and into the direction of the tire, typically being the braking force, referred to as the longitudinal force $F_{L, L}$.

- Force acting parallel to the contact surface and into the direction of the tire spinning axis, referred to as the lateral force $F_{L, Q}$.

\section{Normal Force}

By using the equilibrium of forces in the vertical direction, one can obtain the normal force acting between the tire and the runway surface $F_{T}$ :

$$
F_{T}=k \cdot\left(z-z_{0}\right)+c \cdot \dot{z}+m_{L} \cdot \ddot{z}+m_{L} \cdot g
$$

where $z_{0}$ represents the position of $z$ in which the spring is completely relaxed. However, it has to be noted that since it is a contact condition, the contact force cannot be smaller than zero, i.e. the runway surface cannot pull the wheel downwards. This is important for conditions either when the aircraft leaves the ground or if the runway surface is not even. When the runway surfaces suddenly goes down, depending 
on the landing gear characteristics and the aircraft behavior, the tire could loose ground contact for a short period of time.

The force in normal direction acting on the aircraft frame $F_{L, N}$ can be obtained by considering the equilibrium of forces for the entire landing gear strut:

$$
F_{L, N}=F_{T}-m_{L} \cdot g=k \cdot\left(z-z_{0}\right)+c \cdot \dot{z}+m_{L} \cdot \ddot{z}
$$

\section{Longitudinal Force}

The longitudinal force mainly consists of the braking force that the aircraft uses to slow down after touchdown. Roll friction can also be taken into account, particularly for the nose gear which typically does not have braking systems installed. For the modeling of the braking force, the Magic Formula developed by Pacejka ${ }^{7}$ is used. The Magic Formula provides a relationship between the slip of the tire $s$ and the longitudinal friction coefficient $\mu_{L}$ :

$$
\mu_{L}=D \cdot \sin (C \cdot \arctan (B \cdot(1-E) \cdot s+E \cdot \arctan (B \cdot s)))
$$

with the slip defined as follows:

$$
s=\frac{V_{G S}-V_{W}}{V_{G S}}
$$

The slip is, as seen in Eq. (15), the ratio between the difference of the actual groundspeed $V_{G S}$ compared to the wheel speed $V_{W}$ and the groundspeed. One has to be aware of the fact that this type of modeling cannot be used when the aircraft is close to a full stop due to the singularity in the formula caused by division of a very small $V_{G S}$.

The parameters of this model are the coefficients $B, C, D$ and $E$. They can vary depending on the runway state, the wear of the tire, the temperature of the tire as well as the speed. Fig. 2 shows an example curve of the Magic Formula for a dry runway. The typical curve of the Magic Formula shows a significant increase of the friction coefficient when the slip increases. However, a maximum is reached at the optimum slip value $s_{\text {opt }}$ with the curve descending again afterwards. The friction coefficient when the wheel is locked, i.e. when the slip is one, is therefore smaller than the coefficient obtained at the maximum slip, making antiskid controllers necessary for any ground vehicle in order to achieve the best braking performance. When the slip value is small, a linear relationship can be used for approximation.

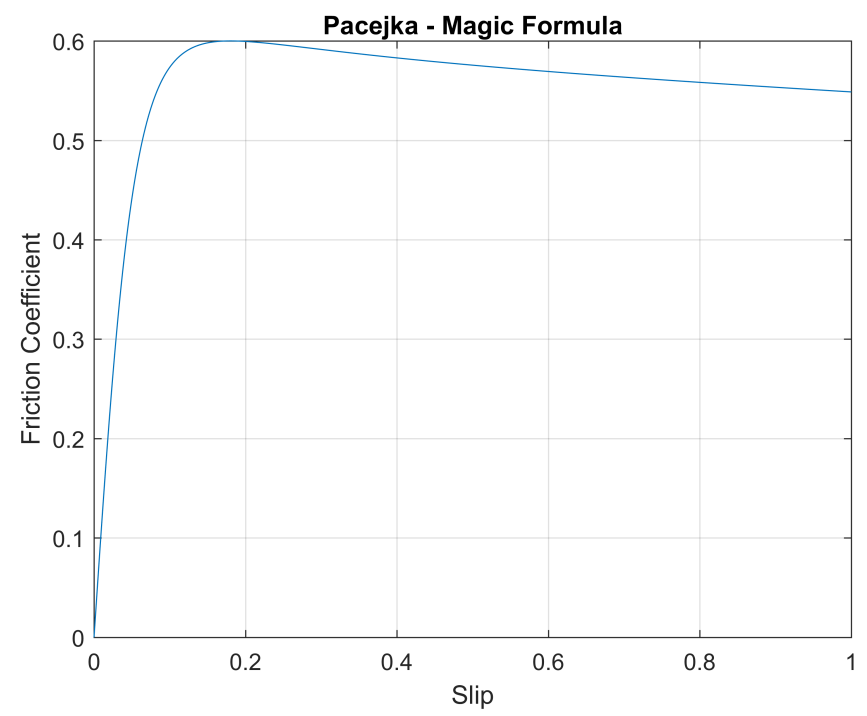

Figure 2. Example curve for the Magic Formula according to Pacejka.

The longitudinal gear force $F_{L, L}$ can then be computed using the normal force and the friction coefficient: 


$$
F_{L, L}=\mu_{L} \cdot F_{L, N}
$$

The slip of the wheel is controlled by the braking system by applying a torque on the wheel via the braking discs. In order to obtain the wheel speed, the rotating part of the wheen and tire, including the braking disc, is considered using the equilibrium of moments.

$$
J \dot{\omega}_{W}=-r_{T} \cdot F_{L, N}+M_{B}
$$

$J$ is the moment of inertia of the entire rotating part, $r_{T}$ is the radius of the tire and $M_{B}$ is the torque applied on the wheel by the braking discs. The wheel speed can be obtained by integrating the wheel angular speed $\omega_{W}$.

$$
V_{W}=\dot{\omega}_{W} \cdot r_{T}
$$

One has to be aware of the fact that when moving to the area after the optimum slip on the slip curve, the behavior of the wheel becomes unstable. Increasing the braking torque will lead to a smaller wheel speed and thus a higher slip. However, a higher slip will result in a lower friction coefficient which further decelerates the wheel. In this case, the wheel will come to a complete stop within a short time frame, meaning that it starts skidding over the runway. This is not a desirable state as the controllability deteriorates and the tire temperature increases significantly, possibly leading to tire bursts. Anti-skid controllers have to be used to encounter this behavior.

\section{Lateral Force}

For the lateral force, the Magic Formula can be used as well. The input variable in this application is not the wheel slip in speed, but rather the sideslip angle between the direction of motion of the tire and the direction of the tire itself, which can be considered as a side slip angle. The Magic Formula will also return friction coefficient values $\mu_{Q}$ that increase with increase side slip, but reach a maximum value for a certain side slip angle. The friction coefficient for a sideslip angle of 90 degrees, i.e. when the wheel is perpendicular to the aircraft moving direction, will be smaller than at the optimum because the wheel is effectively skidding on the runway.

$$
F_{L, Q}=\mu_{Q} \cdot F_{L, N}
$$

\section{Gear Forces and Moments}

To sum it up, the landing gear force for a single gear strut $j$ can be described as follows:

$$
\left(\overrightarrow{\boldsymbol{F}}_{L, j}^{L_{j}}\right)_{O}=\left(k \cdot\left(z-z_{0}\right)+c \cdot \dot{z}+m_{L} \cdot \ddot{z}\right)\left[\begin{array}{c}
-\mu_{L} \\
\mu_{Q} \\
1
\end{array}\right]
$$

The landing gear system of a conventional aircraft typically consist of three landing gear struts: Two main landing gear and one nose gear. The nose gear is typically not equipped with braking devices, but has a steering function to enable lateral control on ground. The total force of the landing gear system can obtained by summation.

$$
\left(\overrightarrow{\boldsymbol{F}}_{L}^{L}\right)_{B}=\sum_{j=1}^{m}\left(\overrightarrow{\boldsymbol{F}}_{L, j}^{L_{j}}\right)_{B}
$$

The moments generated by the landing gear can be obtained by considering the lever arm from the landing gear reference point $j$ to each landing gear $\overrightarrow{\boldsymbol{r}}^{L L_{j}}$ for a total number of $m$ gears.

$$
\left(\overrightarrow{\boldsymbol{M}}_{L}^{L}\right)_{B}=\sum_{j=1}^{m}\left(\overrightarrow{\boldsymbol{r}}^{L L_{j}}\right) \times\left(\overrightarrow{\boldsymbol{F}}_{L, j}^{L_{j}}\right)_{B}
$$




\section{Model Validation}

The validation of the model is performed based on a set of recorded flight data obtained from the Quick Access Recorder (QAR). Model validation, particularly for the aerodynamic components, has already been performed in previous works. ${ }^{6}$ As the aerodynamic coefficients change significantly when the flaps, the landing gear as well as the ground and air spoilers are extended during the approach and landing, the effect of each device should carefully be separated from each other. Therefore, flight phases in which the configuration of the aircraft does not change have to be used to determine the effect of braking.

A very simple relationship is used to describe the motion of the aircraft in the longitudinal direction.

$$
m \cdot \ddot{x}=F_{A, x}+F_{P, x}+F_{L, x}
$$

Only the longitudinal components of the aerodynamic, propulsion and landing gear forces are considered. According to section $\mathrm{B}$, the aerodynamic forces are proportional to the square of the velocity whereas the propulsion forces are proportional to $V^{n_{V}}$, according to section C. The landing gear forces in the longitudinal direction, as described in section $\mathrm{D}$, are dependent on the slip $s$ of the wheel. It is assumed that, due to the deflection of the ground spoilers after landing, the lift coefficient decreases to zero. Thus, the aerodynamics does not influence the normal force of the landing gear.

$$
m \cdot \ddot{x}=F_{A, x}\left(V_{A}^{2}\right)+F_{P, x}\left(V_{A}^{n_{V}}, \omega_{N 1}\right)+F_{L, x}(s)
$$

Using this relationship, five constants, $k_{1}, k_{2}, k_{3}, n_{V}$ and $\mu_{0}$ are introduced by rearranging Eq. (24).

$$
\begin{aligned}
\ddot{x}-\frac{F_{A, x}\left(V_{A}^{2}\right)}{m}-\frac{F_{P, x}\left(V_{A}^{n_{V}}, \omega_{N 1}\right)}{m} & =F_{L, x}(s) \\
\ddot{x}-k_{1} \cdot V_{A}^{2}-k_{2} \cdot \omega_{N 1} \cdot V_{A}^{n_{V}} & =\mu_{0}+k_{3} \cdot s
\end{aligned}
$$

The aerodynamic velocity $V_{A}$ is obtained directly from QAR recodings. Although both calibrated airspeed (CAS) and true airspeed (TAS) are recorded, the CAS should be used as it is directly measured on-board the aircraft. The difference is the use of the reference air density. The CAS assumes the density at Mean Sea Level $\left(\rho_{S}=1.225 \frac{\mathrm{kg}}{\mathrm{m}^{3}}\right.$ ), while the TAS uses the actual density of the air surrounding the aircraft. As the landing phase is examined, the air flow can be considered to be incompressible.

$$
V_{A}=V_{G S}+V_{H W}
$$

The coefficients of the aerodynmic and the propulsion forces are represented by the constants $k_{1}$ and $k_{2}$, respectively. $n_{V}$ is used as an additional constant. $\mu_{0}$ and $k_{3}$ are used to describe the linear relationship. $\mu_{0}$ can be interpreted as the roll friction coefficient, which is small, but will also occur even if there is no slipping tire.

For each set of available flight data, the phase during landing deceleration is used in which the aerodynamic configuration does not change, i.e. the flaps and spoilers are extended. The thrust reverse is active, but a different thrust output is considered by using the rotational speed $\omega_{N 1}$ of the engines.

The left side of Eq. (25) is computed using the available recorded flight data. In order to obtain the longitudinal acceleration, the pitch angle of the aircraft on ground has to be taken into account. The Airbus A340-300, for example, has a nose down pitch angle of 2.4 degrees when loaded with the center of gravity located in the middle, ${ }^{1}$ this value can even slightly increase when braking forces are applied. Therefore, the correction of the longitudinal acceleration using pitch angle measurements has to be performed. On the right side of Eq. (25), the slip value can be computed using Eq. (15). Curve fitting is then performed to obtain the constants $\mu_{0}$ and $k_{3}$ for each flight.

Fig. 3 shows two example flights containing the simulated deceleration caused by the wheels and the measured wheel slip values along with the linear fit according to Eq. (25). For the figure shown on the left, a linear relationship according to the introduced model can be seen in the data. The roll friction value is estimated to be $\mu_{0}=0.003$, which is a realistic value. The maximum friction coefficient that occured during this landing is estimated to be $\mu_{\max }=0.18$. The other parameters are estimated to be: 

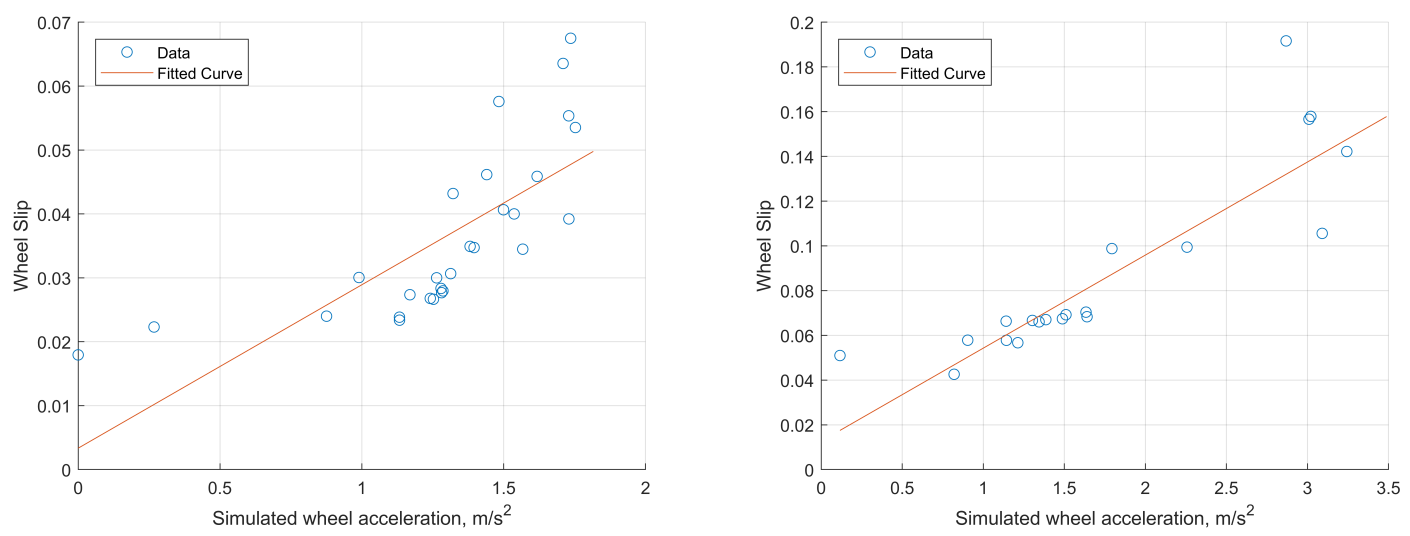

Figure 3. Fitted curve for two example flights.

$$
\begin{aligned}
n_{V} & =-0.7 \\
k_{1} & =3.16 \cdot 10^{-4} \\
k_{2} & =1.02 \cdot 10^{-2} \\
k_{3} & =0.026
\end{aligned}
$$

For these values, the deceleration contributed by the aerodynamics at the time of reverser deployment is computed to be $1.64 \frac{\mathrm{m}}{\mathrm{s}^{2}}=0.167 \mathrm{~g}$, equivalent to an aerodynamic drag coefficient of $C_{D}=0.263$. The right figure displays similar values.

\section{Conclusion and Outlook}

A model has been developed that is capable of describing the forces and moments acting on the aircraft, particularly for the phases of a flight when the aircraft is on the ground. The focus of the model has been on the wheel forces which consists of three components: The vertical component, which mainly supports the weight of the aircraft; the longitudinal component, which mainly consists of the braking forces and the lateral component, which is the traction force keeping the aircraft's direction movement parallel to the longitudinal body axis.

A validation of the model with recorded flight data from the Quick Access Recorder was performed. The results obtained are realistic and are in line with the data, although not all of the examined flights show the behavior described by the model. This can be explained by several factors:

Measurement errors: The measured data certainly contain measurement errors which will also falsify the output from the model.

Low recording frequency: The recording frequency of some parameters in QAR are somewhat low. While this does not apply to all parameters. The longitudinal acceleration, for example, is recorded with 4 $\mathrm{Hz}$. However, the wheel speed, for instance, is recorded only with $0.5 \mathrm{~Hz}$.

As the next step, data enhancement techniques shall be applied. The data can be interpolated in order to increase the available frequency. Furthermore, computed parameters, i.e. parameters that are recorded, but actually computed on-board the aircraft, can be enhanced as well to reduce errors. The groundspeed of the aircraft, for example, can be obtained using estimation techniques. 


\section{Acknowledgments}

This project, Future Sky Safety - P4: Total System Risk Assessment, has received funding from the European Union's Horizon 2020 Research and Innovation Programme under Grant Agreement No 640597.

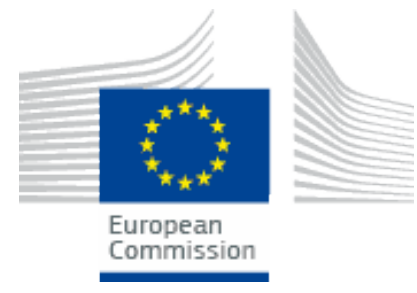

\section{References}

${ }^{1}$ Airbus S.A.S, Aircraft Characteristics: Airport and Maintenance Planning, A340-200/-300, 2017, Airbus S.A.S. Customer Services, Toulouse, France.

${ }^{2}$ Boeing Commercial Airplanes, Statistical Summary of Commercial Jet Airplane Accidents, Worldwide Operations 19592015, 2015, Boeing Commercial Airplanes, Seattle, WA, United States.

${ }^{3}$ International Civil Aviation Organization, Safety Management Manual - Third Edition, DOC 9859, 2013, International Civil Aviation Organization, Montreal, Quebec, Canada.

${ }^{4}$ C. Wang, L. Drees, N. Gissibl, L. Höhndorf, J. Sembiring, F. Holzapfel, Quantification of Incident Probabilities Using Physical and Statistical Approaches, 6th International Conference on Research in Air Transportation, 2014.

${ }^{5}$ C. Wang, L. Drees and F. Holzapfel, Incident Prediction using Subset Simulation, 29th Congress of International Council of the Aeronautical Sciences, 2014

${ }^{6}$ L. Drees, H. Haselhofer, J. Sembiring and F. Holzapfel, Modeling the Flare Maneuver Performed by Airline Pilots Using Flight Operation Data, AIAA Modeling and Simulation Technologies, Boston, 2013.

${ }^{7}$ Bakker, E., Nyborg, L., Hans B. Pacejka, Tyre modelling for use in vehicle dynamics studies, January 1987 Jan, Society of Automotive Engineers, Warrendale, PA. 